Fecha de recepción: 06 de marzo de 2020

Fecha de aceptación:11 de mayo de 2020

DOI:10.19053/01228420.v17.n2.2020.10979
Yenny Catherin Porras Merchán • 1 María Camila Pedreros Benavides $\cdot 2$ Wendy Lorena Reyes Ardila • 3 Helber Enrique Balaguera-López• 4

\title{
Resumen
}

La champa (Campomanesia lineatifolia R. \& P.) es una especie frutal de la familia Myrtaceae, con gran potencial de comercialización por el agradable sabor de sus frutos. Su propagación por semillas presenta dificultades asociadas a un bajo porcentaje de germinación y un tiempo prolongado para culminar esta etapa. Como se desconoce el efecto que pueda generar la luz en las semillas de champa, el objetivo de este estudio fue determinar el efecto de diferentes tratamientos de luz sobre la germinación de semillas champa en condiciones de laboratorio. El material vegetal fue recolectado en el municipio de Miraflores, Boyacá, Colombia. Se hizo un experimento en un diseño completamente al azar con cuatro tratamientos correspondientes a diferentes condiciones de luz: las semillas fueron sembradas en cajas Petri que se cubrieron con papel aluminio (oscuridad), papel celofán rojo, papel celofán azul y un grupo testigo sin cubierta. Cada tratamiento tuvo cuatro repeticiones, la unidad experimental estuvo compuesta de una caja Petri con 35 semillas. Se determinaron parámetros de germinación y de viabilidad a través del test de tetrazolio. Máximo porcentaje de germinación (57,85 \%) y mayor velocidad de germinación (0,39 semillas germinadas/d) con el tratamiento de luz roja; respuesta contraria con las semillas en oscuridad. Las semillas recién cosechadas presentaron 93,74\% de viabilidad. Las que no germinaron obtuvieron un porcentaje representativo de semillas viables (entre el 15 y $20 \%$ ). Estos resultados permiten concluir que $C$. lineatifolia tiene comportamiento de fotoblastismo positivo y presenta condiciones de latencia, posiblemente fisiológica.

Palabras clave: viabilidad, latencia fisiológica, fitocromo, fotoblastismo.

\section{Light Effect on Germination of Champa}

\section{(Campomanesia lineatifolia R. \& P.) seeds}

\section{Abstract}

Champa (Campomanesia lineatifolia R. \& P.), is a fruit species of the Mirtaceae family with high trading potential due to the palatability of its fruits. Its spread by seeds has difficulties related to a low germination percentage and a long time to complete this stage. Taking into account that the effect of the light on champa seeds is unknown, the objective of this study was to determine the effect of different light treatments on the germination of champa seeds under laboratory conditions. The plant material was collected in Miraflores, a municipality of Boyacá state in Colombia. An experiment was carried out in a completely randomized design with 4 treatments corresponding to different light conditions, for which the seeds were sown in Petri dishes that were covered with aluminum foil (dark), red cellophane paper, blue cellophane paper and one group was not covered (control). Each treatment had 4 repetitions, the experimental unit was composed of a Petri dish with 35 seeds. Germination and viability (tetrazolium test) parameters were determined. The maximum germination percentage $(57.85 \%)$ and the highest germination speed ( 0.39 germinated seeds/d) were obtained with the red light treatment, the opposite response was obtained with the seeds in the dark. The freshly harvested seeds presented $93.74 \%$ viability. The seeds that did not germinate had a representative percentage of viable seeds (between 15 and $20 \%$ ). These results allow us to conclude that C. lineatifolia has a positive photoblastism behavior and presents dormancy conditions, possibly physiological dormancy.

\section{Keywords:viability, physiological dormancy, phytochrome, photoblastism.}

1. Ingeniero agrónomo, independiente. Tunja, Boyacá, Colombia. caterinsnup@gmail.com. (D) http://orcid.org/0000-0003-3764-0354 2. Ingeniero agrónomo. Universidad Pedagógica y Tecnológica de Colombia. Tunja, Boyacá, Colombia. mariacamila.pedreros@uptc.edu.co. (1D) http://orcid.org/0000-0002-2813-2238

3. Ing. Agrónomo. Universidad Pedagógica y Tecnológica de Colombia. Tunja, Boyacá, Colombia. wendy.reyes@uptc.edu.co. (D)http://orcid.org/0000-0003-2946-0273 4. Ph.D. Profesor asociado. Universidad Nacional de Colombia. Bogotá D. C. Colombia. hebalagueral@unal.edu.co. (D) http://orcid.org/0000-0003-3133-0355 


\section{I.Introducción}

La champa (Campomanesia lineatifolia R. \& P.) pertenece al género Campomanesia de la familia Myrtaceae (Parra-0, Bayly, Drinnan, Udovicic \& Ladiges, 2014). En Colombia es cultivada principalmente en el departamento de Boyacá, en la provincia de Lengupá (Balaguera, Álvarez \& Bonilla, 2009). Con el fruto se pueden elaborar productos frescos y procesados de forma similar a como ocurre con frutos de otras mirtáceas como la guayaba (Parra, 2014) y la feijoa (Parra \& Fischer, 2013). Debido a su exótico sabor, la champa tiene un alto potencial de comercialización y recientemente se está introduciendo en los mercados de Colombia (López \& Rodríguez, 1995; Balaguera et al., 2009; Balaguera, Herrera \& Cortés, 2012), lo cual resalta la importancia de introducir nuevas especies frutales al mercado de este país, como ocurre con otras especies (Arias, Velásquez, Mateus, Chaparro \& Orduz, 2016). El fruto posee de 6 a 8 semillas de $1 \mathrm{~cm}$ de diámetro aproximadamente, que representan un porcentaje importante del tamaño del fruto (Álvarez, Galvis \& Balaguera, 2009; Balaguera et al., 2012). Estas semillas, al parecer, poseen un alto y diverso contenido de metabolitos secundarios (Bonilla et al., 2005; Osorio et al., 2006). Los compuestos extraídos de la semilla tienen actividad antimicrobial y supuestamente contribuyen a expresar los aromas y sabores característicos de la champa en todos los estadios de crecimiento del fruto, y se ha demostrado que también tienen actividad bioherbicida (Salinas, 2016; Martínez, 2019).

Actualmente, la champa se propaga por vía sexual a través de semillas. Sin embargo, se tienen problemas con la propagación a escala comercial por los bajos porcentajes de germinación y el prolongado tiempo requerido para obtener una planta adecuada para el trasplante. Una posible causa de este problema puede estar asociada a algún tipo de latencia, es decir, que incluso si la semilla se encuentra en condiciones favorables, no germina (Hartmann, Kester, Davies \& Geneve, 2011; Bewley, Bradford, Hilhorst \& Nonogaki, 2013). Los frutales tropicales como la champa sufren un proceso incipiente de domesticación, debido a que muchas de estas especies han pasado de ser plantas silvestres a cultivadas, con un síndrome de la domesticación incompleto (Kawasaki \& Pérez, 2012) que les permite conservar las condiciones de latencia. En este sentido, se considera importante conocer las condiciones apropiadas que permitan una óptima germinación de la semilla.

Dentro de las condiciones necesarias para que ocurra la germinación están el oxígeno, la temperatura, el agua y la luz (Baskin \& Baskin, 2014; Bewley et al., 2013). Este último factor es un estímulo que puede ser percibido por fotorreceptores como los fitocromos, que tienen la posibilidad de captar diferentes longitudes de onda e inducir cambios en la expresión génica, que conducen a activar la germinación (Hartmann et al., 2011; Bewley et al., 2013). Varias especies de mirtáceas pueden presentar algún tipo de fotolatencia y necesitar varias horas de luz diaria para germinar (Ortega et al., 2011).

En especies de la familia Myrtaceae se reporta la aplicación de tratamientos pregerminativos para eliminar condiciones de latencia, como hidroacondicionamiento, aplicación de giberelinas, entre otros, pues se ha reportado la presencia de latencia morfológica y morfofisiológica (Rego, Cosmo, Gogosz, Kuniyoshi \& Nogueira, 2011; Latsague, Sáez \& Coronado, 2010). Por tanto, el objetivo de este estudio fue evaluar el efecto de la luz sobre la germinación de semillas de champa (Campomanesia lineatifolia R. \& P), bajo condiciones de laboratorio, con el fin de optimizar el proceso de propagación de esta especie.

\section{Materiales y métodos}

Los frutos de champa utilizados en el estudio se cosecharon manualmente en estado de madurez de consumo (60\% amari$110-40 \%$ verde claro) (Álvarez et al., 2009), con un tamaño homogéneo. Estos provenían de árboles de aproximadamente 15 años de edad, del municipio de Miraflores (departamento de Boyacá), ubicado a 1.432 m.s.n.m, latitud $5^{\circ} 11^{\prime}$ y longitud $73^{\circ} 08^{\prime}$, el cual presenta una precipitación promedio de 2500 $\mathrm{mm}$ y una temperatura que oscila entre los 18 y $24^{\circ} \mathrm{C}$. Los experimentos se llevaron a cabo en el Laboratorio de Fisiología Vegetal de la Universidad Pedagógica y Tecnológica de Colombia, con condiciones de temperatura promedio de $18 \pm 2{ }^{\circ} \mathrm{C}$, humedad relativa del $78 \%$ y fotoperiodo de $12 \mathrm{~h}$.

Las semillas se extrajeron manualmente; después se lavaron y se dejaron secar al ambiente durante dos horas. Al cabo de este tiempo se realizó la siembra en cajas Petri con papel filtro como sustrato. Se empleó un diseño completamente al azar con cuatro tratamientos correspondientes a diferentes condiciones de luz, para lo cual las semillas de champa fueron sembradas en cajas Petri cubiertas de acuerdo con los tratamientos: papel aluminio (T1), papel celofán rojo (T2), papel celofán azul (T3), testigo sin cobertura (T4). Cada tratamiento tuvo cuatro repeticiones, la unidad experimental fue una caja Petri con 35 semillas. Se regó a diario con agua destilada para mantener húmedo el papel filtro y garantizar el proceso de imbibición de las semillas.

La primera lectura de germinación se hizo a los 15 días después de la siembra, y se siguió realizando cada tercer día, durante 64 días después de la siembra, hasta que se obtuvo germinación 
constante. Se tuvo como criterio de germinación que la radícula midiera mínimo $5 \mathrm{~mm}$. A partir de estos datos y con las fórmulas utilizadas por Carranza, Castellanos, Deaza y Miranda (2016) y propuestas por Ranal y Santana (2006) se calcularon: el porcentaje de germinación, la velocidad media de germinación (semillas germinadas/d) y el tiempo medio de germinación (d).

Las semillas que no germinaron en cada uno de los tratamientos se sometieron a la prueba de viabilidad mediante el test de tetrazolio, para lo cual a cada semilla se le hizo un corte longitudinal con un bisturí, luego se colocaron dentro de cajas de Petri en una solución de cloruro de 2,3,5 trifenil tetrazolio $(0,5$ $\%)$, y se dejaron durante 2 horas a una temperatura de $37^{\circ} \mathrm{C}$. Cumplido este tiempo se hizo evaluación visual de la tinción de acuerdo con las indicaciones de la International Seed Testing Association (ISTA, 2019).

Con el fin de conocer la viabilidad inicial de las semillas, se seleccionaron 200 semillas recién extraídas del fruto y se dividieron en cuatro grupos, se imbibieron durante 8 horas $y$ luego se siguió el mismo protocolo de tinción con tetrazolio indicado previamente.

Con los datos obtenidos se realizaron pruebas de Shapiro-Wilk (normalidad de los residuales) y Levene (homogeneidad de varianzas); posteriormente se llevaron a cabo análisis de varianza, y cuando hubo diferencias significativas $(p<0,05)$ se realizó una prueba de comparación de promedios de DMS ( $P \leq$ $0,05)$. Los análisis se hicieron con el programa estadístico SPSS v.19.

\section{Resultados y discusión}

\section{A. Porcentaje de germinación}

Se presentaron diferencias estadísticas significativas $(P<0,05)$, donde el tratamiento con cobertura roja tuvo el mayor porcentaje de germinación (57,85 $\pm 2.22 \%$ ); situación contraria se observó con el tratamiento sin luz, donde la germinación fue menor $(45,71 \pm 0.90 \%)$. Al evaluar el porcentaje de semillas germinadas se constató que no existe diferencia significativa entre los tratamientos con luz, lo cual indica que en el proceso de germinación la luz (blanca, azul o roja) representa un factor determinante (Figura 1). Estos resultados concuerdan con los encontrados en otras especies de la familia Myrtaceae, como Campomanesia guazumifolia, Myrcianthes pungens y Psidium cattleyanum, también fueron fotoblásticas positivas (Filho, 2015).

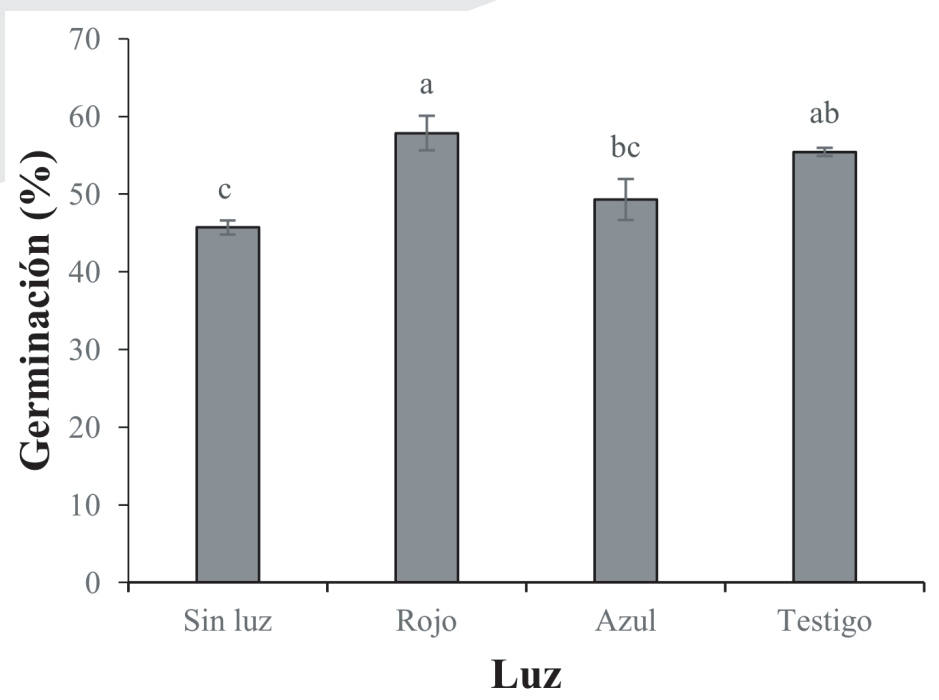

Figura 1. Efecto de la luz sobre el porcentaje de germinación de semillas de champa (Campomanesia lineatifolia R. \& P). Promedios seguidos de letras diferentes presentan diferencias estadísticas de acuerdo con la prueba de DMS $(p<0,05)$. Las barras verticales en cada promedio indican el error estándar.

El espectro de luz juega un papel importante en la germinación y el crecimiento de las plántulas (Li, Xu, Liu \& Han, 2012). Según Lima, Goldenberg y Sobral (2015), la calidad de luz y diferentes reguladores de crecimiento intervienen en el proceso de germinación. La luz es un estímulo determinante debido a la gran cantidad de fotorreceptores que poseen las semillas (Bewley et al. 2013), por ello, las semillas fotoblásticas positivas presentan mayor porcentaje de germinación en presencia de luz (Bewley et al., 2013; Hartmann et al., 2011), similar a lo encontrado en las semillas de champa, lo que permite inferir que estas semillas tienen un comportamiento fotoblástico positivo. Resultados similares fueron encontrados por Lamarca, Silva y Barbedo (2011), quienes reportaron respuesta favorable a la luz en semillas de otras Myrtaceaes como Eugenia brasiliensis, E. involucrata, E. pyriformis y E. uniflora. Por su parte, Filho (2015) informó que la germinación de semillas de Blepharocalyx salicifolius (Myrtaceae) aumentó cuando se trató con luz roja, debido posiblemente a que los fitocromos son más abundantes en tejidos jóvenes (Taiz, Zeiger, Moller \& Murphy, 2017) y explicaría el efecto favorable que tuvo este tipo de luz en la germinación de semillas de champa.

En semillas de E. involucrata (Takaki, 2015) y de Campomanesia aurea (Emer, Paolazzi, Fior \& Schafer, 2018), la germinación fue favorecida por el uso de filtros rojos; sin embargo, con filtros azules la germinación fue menor. Estos están parcialmente de 
acuerdo con los encontrados en champa, donde la germinación fue inhibida parcialmente por la luz azul. No obstante, los valores obtenidos fueron similares a las semillas que permanecieron sin luz (Figura 1). Esto resulta importante, ya que no existe información sobre germinación para esta especie y mucho menos para sus requerimientos lumínicos. Los requisitos lumínicos para la germinación en diferentes especies están relacionados con los grupos ecológicos a los que pertenecen. Por lo tanto, en condiciones de sombra, donde predomina la radiación roja distante, las semillas no germinan, ya que se considera un ambiente no propicio para el desarrollo de plántulas debido a una baja radiación azul y roja (Takaki, 2015).

Cabe señalar que la germinación fue inferior al $90 \%$, lo que indica que hay otros factores que están afectando la germinación de las semillas de champa. Al respecto, Filho (2016) indica que características como la integridad morfológica, estructural y las condiciones de latencia pueden afectar el porcentaje y la velocidad de germinación.

\section{B. Velocidad media de germinación (VGM)}

Se presentaron diferencias significativas entre los tratamientos de luz $(p<0,05)$. Los resultados indicaron que el tratamiento que mostró mayor velocidad de germinación fue el de la luz roja, con 0,39 0.02 semillas germinadas/d; por el contrario, el menos efectivo fue el de oscuridad, con $0,30 \pm 0,0007$ semillas germinadas/d (Figura 2A).

Se observó un efecto positivo de la luz en la velocidad de germinación, lo cual confirma lo que mencionan Camargo et al. (2015), quienes indican que semillas con esta característica se consideran como fotoblásticas positivas. Es así que las condiciones de luz tienen un efecto sobre la velocidad de germinación (Wang \& Folta, 2013). Las semillas poseen fotorreceptores tipo fitocromos que tienen como función captar diferentes longitudes de onda, en este caso longitudes del rojo; como consecuencia se genera un proceso de señalización celular que induce la síntesis de giberelinas (Hartmann et al., 2011; Bewley et al., 2013). Esta hormona tiene incluida la activación de enzimas hidrolíticas y reacciones de fotorreceptores que activan la germinación (Savaedi, Parmoon, Moosavi \& Bakhshande, 2019); como consecuencia, la germinación puede ser mayor y puede ocurrir a mayor velocidad, tal como se presentó en este estudio.

Desde el punto de vista práctico, germinar las semillas a libre exposición o utilizar una cobertura de color rojo, que simulen el efecto de la longitud de onda roja, pueden ser opciones viables para optimizar el proceso de obtención de plántulas de champa.

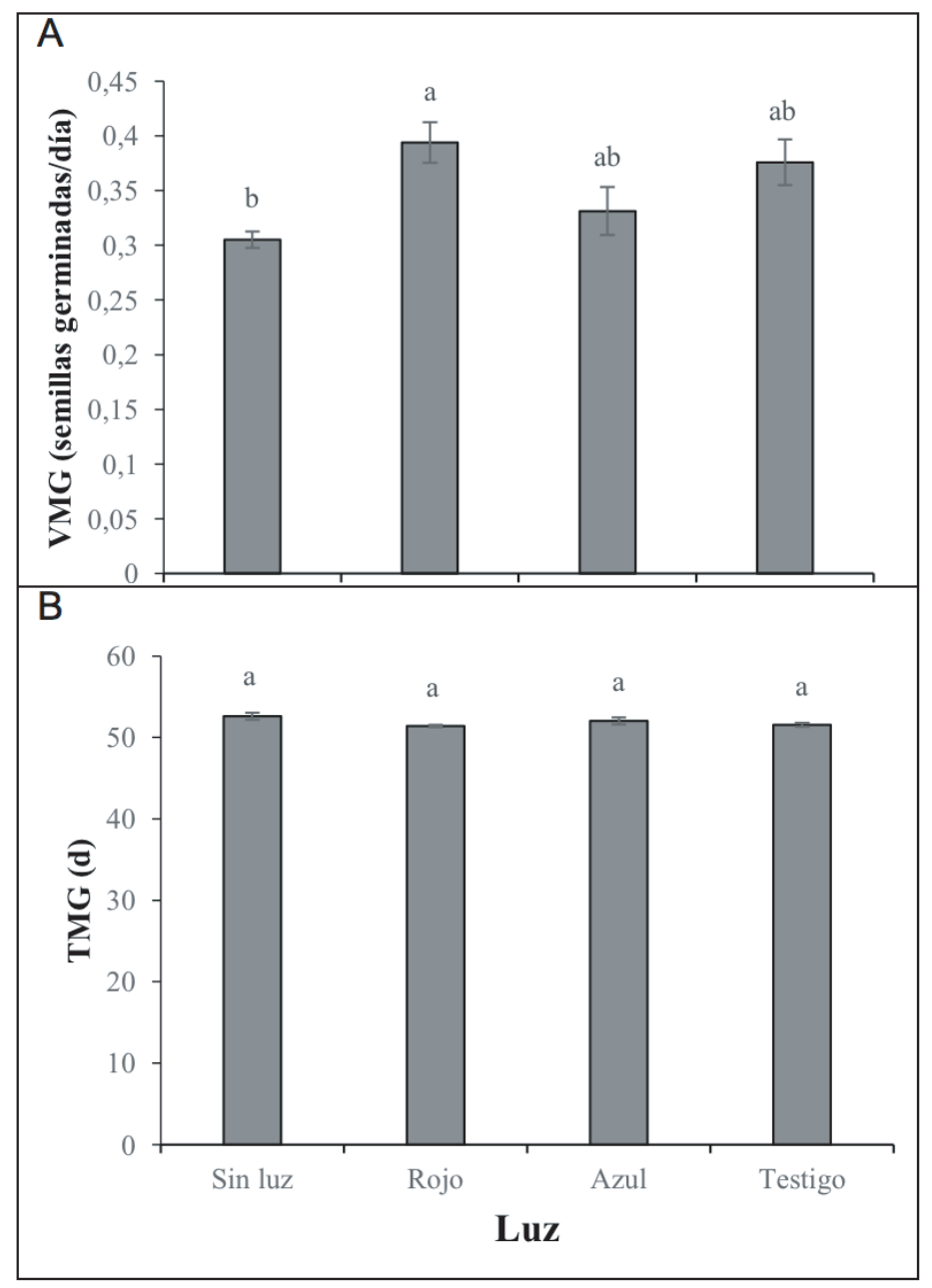

Figura 2. Efecto de la luz sobre A) la velocidad media de germinación y B) el tiempo medio de germinación de semillas de champa (Campomanesia lineatifolia R. \& P). Promedios seguidos de letras diferentes presentan diferencias estadísticas de acuerdo con la prueba de DMS $(p<0,05)$. Las barras verticales en cada promedio indican el error estándar.

\section{Tiempo medio de germinación (TMG)}

No se encontraron diferencias estadísticas $(p>0,05)$. Se observó un tiempo promedio en el experimento de 52 días (Figura 2B). Este tiempo se considera alto e indica que se debe seguir estudiando la fisiología de esta especie para reducirlo y hacer más eficiente el proceso de propagación de la champa.

El hecho de que el TMG no presentara diferencias significativas está asociado a que en los tratamientos con mayor germi- 
nación, el mayor número de semillas germinadas requiere también un tiempo considerable para llevar a cabo este proceso. En el caso de las semillas sin luz, estas tardaron estadísticamente el mismo tiempo en germinar, pero lograron un menor porcentaje de germinación. Este resultado igualmente pone en evidencia que la luz en estas semillas no es un requerimiento absoluto para inducir la germinación, ya que sin luz también un porcentaje importante de semillas lo puede lograr y en un tiempo similar a las semillas de los demás tratamientos. Esto indica que hay otros factores que también son determinantes en el proceso de germinación, en los que se incluye la disponibilidad de agua y oxígeno $y$, por supuesto, la temperatura, y su interacción puede promover diferenciación en la respuesta fotoblástica entre poblaciones (Castillo et al., 2013). Teniendo en cuenta lo anterior, todavía son muchos los aspectos de la germinación de esta especie que se desconocen. Pero asimismo se puede pensar que en la champa el poco tiempo de domesticación hace que esta especie pueda germinar en un amplio rango de condiciones de luz, así el porcentaje de germinación sea bajo.

\section{Viabilidad de las semillas}

En cuanto a la categorización de la viabilidad inicial de las semillas, se encontró un alto porcentaje de semillas viables $(93,74 \pm 1,11 \%)$ antes de iniciar el proceso de germinación (Figura 3). Si se tiene en cuenta que la germinación hallada fue inferior a la viabilidad, hay que indicar que están ocurriendo dos situaciones. La primera tiene que ver con una condición de latencia en las semillas, y la segunda, con la muerte de un porcentaje de las semillas. Estas situaciones se corroboran con los resultados de la prueba de viabilidad reportada para cada uno de los tratamientos de luz (figuras 4 y 5), donde a pesar de que no hay diferencias estadísticas entre los tratamientos de luz para las semillas viables, este porcentaje está entre el 15 y el 20 \% (Figura 4). En el caso de las semillas no viables, los resultados son interesantes, porque se presenta un alto porcentaje de semillas (superior al $20 \%$ ) que finalmente murieron. Pero este porcentaje es significativamente inferior en el tratamiento de luz roja, lo cual indica el efecto positivo que tiene este tipo de luz en la fisiología de la germinación de las semillas de champa, posiblemente mediante la activación del fitocromo que induce señalización para inhibición de síntesis de $A B A$ y promoción de la síntesis de GA (Bewley et al., 2013). Y el mayor porcentaje de semillas no viables $(37 \pm 6 \%$ ) se encontró en las semillas germinadas en oscuridad (figuras 4 y 5 ).

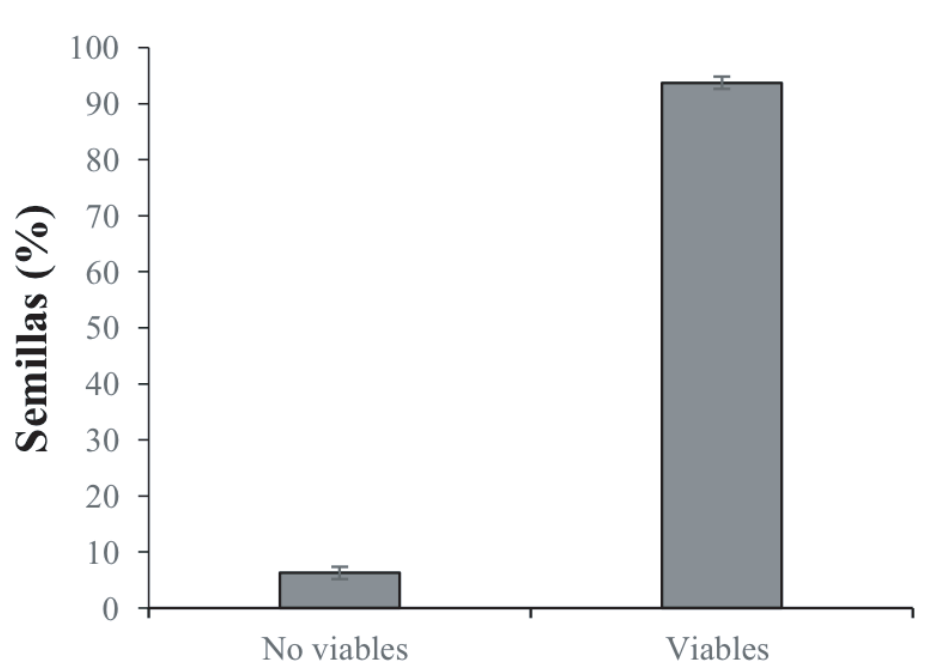

Categoría de las semillas

Figura 3. Categorización de la viabilidad de semillas de champa (Campomanesia lineatifolia R. \& P.) mediante test con tetrazolio. Las barras verticales en cada promedio indican el error estándar $(n=4)$

La prueba de tetrazolio ofrece una rápida determinación de la viabilidad de las semillas, y es de gran importancia en las especies que presentan latencia (Gadotti \& Villela, 2016). Los resultados permitieron calcular que los frutos utilizados contenían semillas latentes, puesto que el porcentaje de semillas viables obtenidas en la prueba de tetrazolio es mayor que el porcentaje de semillas germinadas conseguidas en el ensayo de germinación. Baskin y Baskin (2014) indicaron que las semillas de muchas especies exhiben latencia en el estado de madurez completa de los frutos y que no presentan germinación en un conjunto amplio de condiciones ambientales. Esto corrobora el hecho de que cada especie es diferente y que la calidad de la semilla cambia según los factores internos y externos (Maldonado et al., 2016), refiriéndose así a la variación en el tamaño y los estados de maduración de las semillas dentro de un fruto (Mancipe, Calderón \& Pérez, 2018), los factores ambientales involucrados y los relacionados con la viabilidad y vitalidad, longevidad, grado de madurez y genotipo (Filho, 2016). 


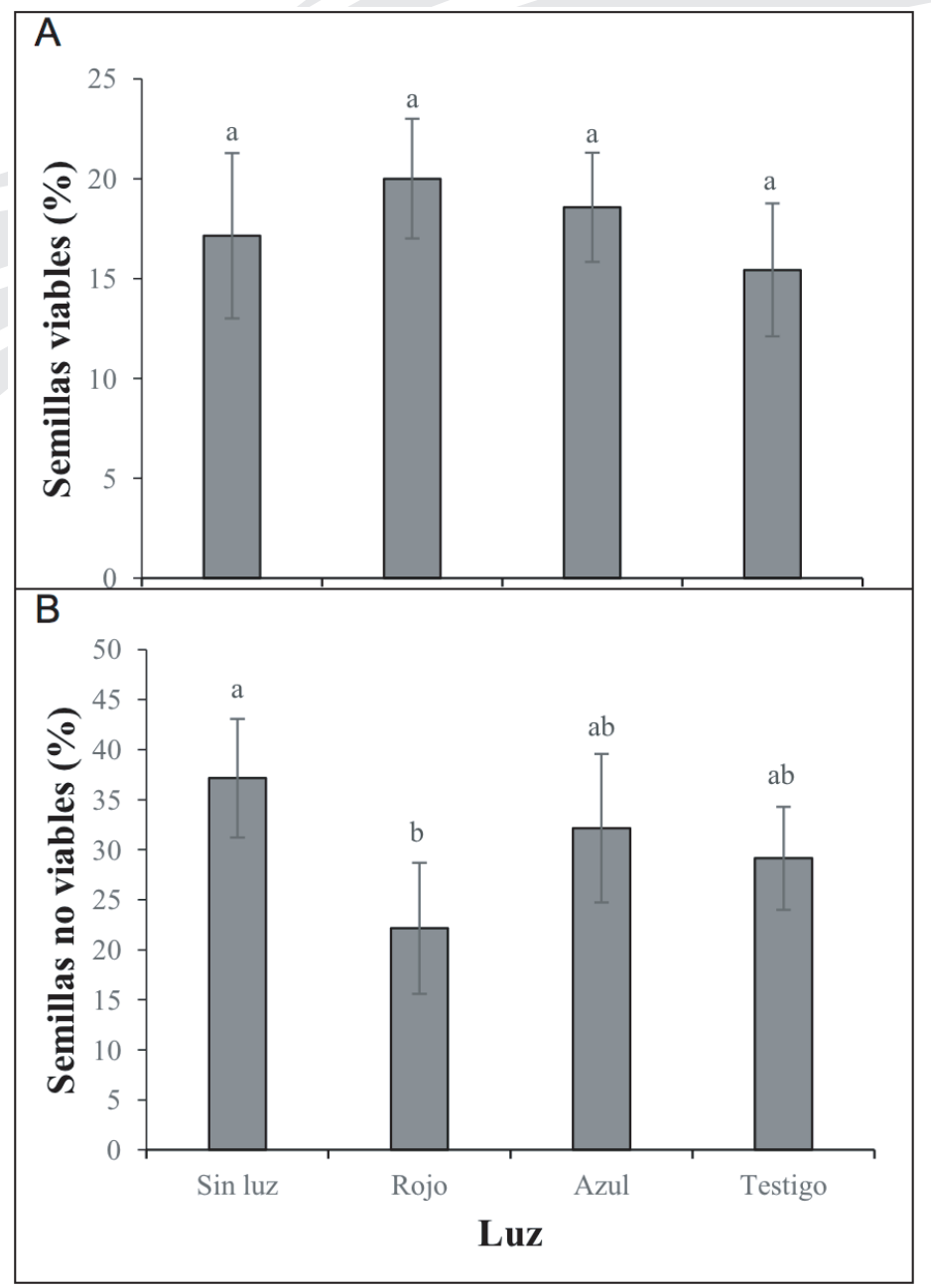

Figura 4. Porcentaje de semillas viables A) y porcentaje de semillas no viables B) de champa (Campomanesia lineatifolia R. \& P) sometidas a diferentes tipos de luz. Promedios seguidos de letras diferentes presentan diferencias estadísticas de acuerdo con la prueba de DMS $(p<0,05)$. Las barras verticales en cada promedio indican el error estándar $(n=4)$

Las semillas, después de ser expuestas a las coberturas de colores, lograron germinar; aunque dejamos en claro que los factores de luz u oscuridad pueden ser requeridos para la germinación o pueden provocar su inhibición. Por ello, al observar que la luz incide positivamente sobre la germinación de las semillas de champa, se puede corroborar que estas presentan indicios de latencia fisiológica dependiente de la luz (Salazar \& Cancino, 2012). Al respecto se ha indicado que, en condiciones espontáneas, las plantas tienen que responder a condiciones variables, en tiempo y espacio, constituyendo la latencia una estrategia para dispersar la germinación en tiempo (Delahaie et al., 2013). Algunos estudios señalan que los factores ambientales, como la precipitación, la temperatu- ra y la irradiancia, afectan la planta madre y la calidad de las semillas en desarrollo, lo que repercute en la capacidad germinativa de las semillas (Baradwaj, Rao, Baskin \& Kumar, 2016). La latencia es un fenómeno influenciado por la información genética de la especie, las condiciones ambientales, el balance de reguladores del crecimiento, como ácido abscísico y giberelinas, y la interacción de estos dos con el etileno, y algunas auxinas o brasinoesteroides (Baskin \& Baskin, 2014; Taiz et al., 2017). La latencia puede estar relacionada con el embrión o con las estructuras que lo rodean, como la testa, lo que permite pensar en varias clases de latencia (Finch \& Leubner, 2006) que estarían aún sin dilucidar para las semillas de champa.

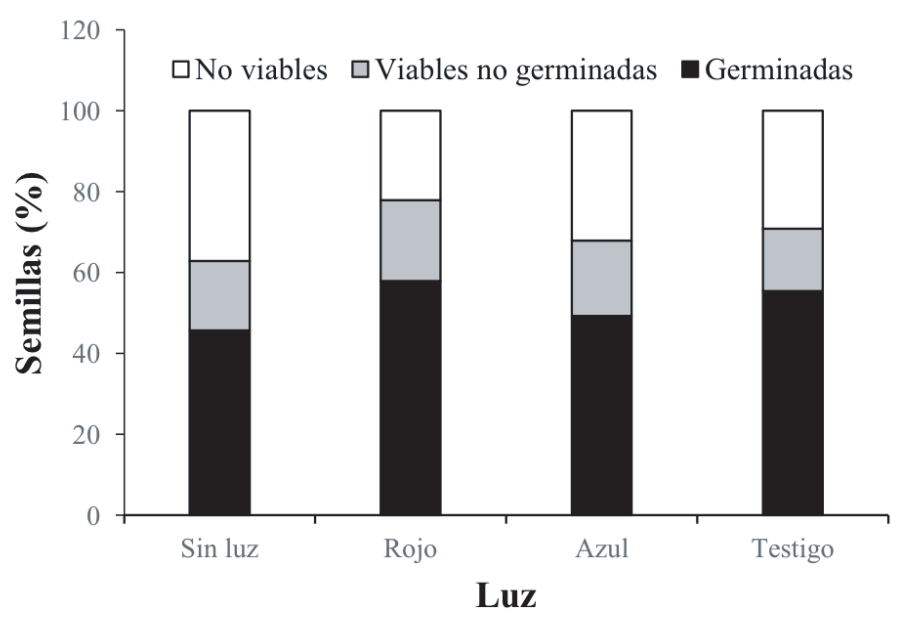

Figura 5. Efecto del color de la cobertura sobre el porcentaje de semillas viables, no viables y germinadas de champa (Campomanesia lineatifolia R. \& P).

Por último, si las semillas tienen pocas reservas o están metabólicamente muy activas para soportar períodos largos antes de la germinación, la inactividad prolongada conduciría a su muerte (Donohue et al., 2010). En este contexto, las características del banco de semillas y la longevidad de estas no se han documentado en Campomanesia lineatifolia, lo que confirma la importancia de continuar evaluando las poblaciones de esta especie endémica.

\section{Conclusiones.}

Los resultados permiten concluir que la luz influyó positivamente en el proceso de germinación de la champa (Campomanesia lineatifolia R. \& P), principalmente la luz de color rojo, lo cual revela la condición de fotoblastismo positivo de esta especie. Además, las semillas de champa mostra- 
ron porcentajes de viabilidad superiores a los porcentajes de germinación que indican la presencia de latencia, que al parecer es fisiológica, porque las semillas germinaron en mayor porcentaje en presencia de luz. Lo anterior sugiere que se deben realizar más estudios para entender los mecanismos que están regulando la latencia fisiológica en esta especie y las estrategias para incrementar su germinación en un menor tiempo.

\section{Referencias}

Álvarez, J. G., Galvis, J. A. \& Balaguera, H. E. (2009). Determinación de cambios físicos y químicos durante la maduración de frutos de champa (Campomanesia lineatifolia R. \& P.). Agronomía Colombiana, 27, 253-259.

Arias, M. H., Velásquez, D., Mateus, H. Chaparro, Z. \& Orduz-Rodríjuez, J. (2016). El rambután (Nephelium lappaceum), frutal asiático con potencial para Colombia: avances de la investigación en el piedemonte del Meta. Revista Colombiana de Ciencias Hortícolas 10(2), 262-2. Doi: http://dx.doi.or$\mathrm{g} / 10.17584 / \mathrm{rcch} .2016 \mathrm{v} 10 \mathrm{i} 2.5761$

Balaguera, H. E, Herrera, A. \& Cortés, D. (2012) Crecimiento de frutos de champa en condiciones agroecológicas de Miraflores, Boyacá, Colombia. Pesquisa Agropecuária Brasileira, 47(12), 1722-1730. Doi: https://doi.org/10.1590/S0100-204X2012001200007

Balaguera, H. E., Álvarez, J. G. \& Bonilla, D. C. (2009). Crecimiento y desarrollo del fruto de champa (Campomanesia lineatifolia Ruiz \& Pavón). Revista UDCA Actualidad y Divulgación Científica, 12(2), 113-123. Doi: https://doi.org/10.31910/rudca.v12.n2.2009.697

Baradwaj, R. G., Rao, M. V, Baskin, C. C. \& Kumar, T. S. (2016). Non-deep Simple Morphophysiological Dormancy in Seeds of the Rare Alpinia galanga: A First Report for Zingiberaceae. Seed Science Research, 26(2), 165-170. Doi: https://doi.org/10.1017/S0960258516000039.

Baskin, C. C. \& Baskin, J. M. (2014). Seeds: Ecology, Biogeography, and Evolution of Dormancy and Germination. (2nd ed.). San Diego, CA: Academic Press.

Bewley, D. J., Bradford, K. J., Hilhorst, H. W. M. \& Nonogaki, H. (2013). Seeds: Physiology of Development, Germination and Dormancy. (Third ed.). Springer Science. 399 Doi: http://dx.doi.org/10.1007/978-1-4614-4693-4
Bonilla, A., Duque, C., Garzón, C., Takaishi, Y., Yamaguchi, K. Hara, N. \& Fujimoto, Y. (2005). Champanones, Yellow Pigments from the Seeds of Champa (Campomanesia lineatifolia). Phytochem. 66, 1736-1740. Doi: https://doi.org/10.1016/j.phytochem.2005.05.025

Camargo, S., Rodrigues, D., Rodrigues, C., De Assis, A., De Faria, R. \& Schuch, M. W. (2015). Fitorreguladores y espectros de luz na micropropagacao de Oncidium baueri Lindl. Ciência Rural, 45(11), 2007-2012. Doi: 10.1590/0103-8478cr20141780

Carranza, C., Castellanos, G., Deaza, D. \& Miranda, D. (2016). Efecto de la aplicación de reguladores de crecimiento sobre la germinación de semillas de badea (Passiflora quadrangularis $L$.) en condiciones de invernadero. Revista Colombiana de Ciencias Hortícolas, 10(2), 284-291. Doi: https://doi.org/10.17584/rcch.2016v10i2.5791

Castillo, M., Bustamante, O. R., Peña, F. T., Gutiérrez, V. L., Reyes, C. A., Arredondo, A. \& Marey, M. (2013). Negative Photoblastism in the Invasive Species Eschscholzia californica Cham. (Papaveraceae): Patterns of Altitudinal Variation in Native and Invasive Range. Gayana Botanica, 70(2), 330-336. Doi: https://doi.or$\mathrm{g} / 10.4067 / \mathrm{S} 0717-66432013000200010$

Delahaie, J., Hundertmark, M., Bove, J., Leprince, 0., Rogniaux, H. \& Buitink, J. (2013). LEA Polypeptide Profiling of Recalcitrant and Orthodox Legume Seeds Reveals ABI3-regulated LEA Protein Abundance Linked to Desiccation Tolerance. Journal of Experimental Botany, 64(14), 4559-73. Doi: https://doi.org/10.1093/jxb/ert274

Donohue, S., Alvarenga, A., Guimarães, R., Lara, T., Custódio, T. \& Chaves, I. (2010). Ecofisiologia da germinação de sementes de Campomanesia pubescens. Ciência Rural, Santa Maria, 41(8), 1362-1368. Doi: https://doi.or$\mathrm{g} / 10.1590 / \mathrm{S} 0103-84782011000800011$

Emer, A., Paolazzi, J., Fior, C. y\& Schafer, G. (2018). Fotoblastismo e germinação de sementes de Campomanesia aurea (Myrtaceae). Biosci. J. Uberlândia, 34(6), 1505-1512.

Filho, M. (2015). Fisiologia de sementes de plantas cultivadas. Londrina, PR: Abrates.

Filho, M. (2016). Fisiología de semillas de plantas cultivadas. (2a ed.). Londrina, PR: Abrates.

Finch, W. \& Leubner, G. (2006). Seed Dormancy and the 
Control of Germination. New Phytologist, 171, 501-523. Doi: 10.1111 / j.1469-8137.2006.01787.x

Gadotti, G. I. \& Villela, F. A. (2016). Can the Tetrazolium Test be used as an Alternative to the Germination Test in Determining Seed Viability of Grass Species? Crop Science, 56(1), 707-715. Doi: https://doi.org/10.2135/cropsci2015.06.0399

Hartmann, H., Kester, D. E., Davies, F. T. \& Geneve, R. L. (2011). Hartmann \& Kester's Plant Propagation: Principles and Practices. (8th ed.). Upper Saddle River, N.J.: Prentice Hall International.

International Seed Testing Association -ISTA-. (2019). Rules Proposals for the International Rules for Seed Testing. Retrieved from https://www.ingentaconnect.com/content/ista/rules.

Kawasaki, M. L. \& Pérez, A. (2012). A New Species of Plinia (Myrtaceae) from Ecuador, with Demographic Notes from a Large Forest Plot. Harvard Papers in Botany, 17(1), 19-20. Doi: https://doi.org/10.3100/025.017.0105

Lamarca, E., Silva, C. \& Barbedo, C. (2011). Limites térmicos para a germinação em função da origem de sementes de espécies de Eugenia (Myrtaceae) nativas do Brasil. Acta Botanica Brasilica, Belo Horizonte, 25(2), 293-300. Doi: https://doi.org/10.1590/S0102-33062011000200005.

Latsague, M., Sáez, P. \& Coronado, L. (2010). Tratamientos pregerminativos para Myrceugenia exsucca (Myrtaceae). Bosque (Chile) 31(3), 243-246. Doi: http://dx.doi.or$\mathrm{g} / 10.4067 / \mathrm{S} 0717-92002010000300008$

Li, H., Xu, Z., Liu, X. \& Han, X. (2012). Effects of Different Light Sources on the Growth of Non-heading Chinese Cabbage (Brassica campestris L.). Journal of Agricultural Science, 4(4), 262-273. Doi: https://doi.org/10.5539/jas.v4n4p262

Lima, D., Goldenberg, R. \& Sobral, M. (2015). Gênero Campomanesia (Myrtaceae) no estado do Paraná, Brasil. Rodriguésia, Rio de Janeiro, 62(3), 683-693.

López, M. \& Rodríguez, J. (1995). Diagnóstico del mercadeo de la champa en el Municipio de Miraflores Boyacá. (Tesis para optar al título de tecnólogo en Mercadeo). Instituto de Educación Abierta y a Distancia. Universidad Pedagógica y Tecnológica de Colombia, Tunja.

Maldonado, M., García, G., García, J., Ramírez, C., Hernández, A., Valdez, J., Torrez, T. \& Cetina, V. (2016). Seed Viability and
Vigour of two Nanche Species (Malpighia mexicana and Byrsonima crassifolia). Seed Sci \& Technol, 44(1), 1-9. Doi: 10.15258/sst.2016.44.1.03

Mancipe, C., Calderón, M. \& Pérez, V. (2018). Evaluación de viabilidad de semillas de 17 especies tropicales altoandinas por la prueba de germinación y la prueba de tetrazolio. Caldasia, 40(2), 366-382. Doi: http://dx.doi.org/10.15446/caldasia.v40n2.68251.

Martínez, C.A. (2019). Efecto alelopático del extracto de semillas de chamba (Campomanesia lineatifolia) sobre la germinación y crecimiento de plántulas de cerraja (Sonchus oleraceus L.) en condiciones de laboratorio. (Tesis). Universidad Nacional Abierta y a Distancia (UNAD). Escuela de Ciencias Pecuarias y del Medio Ambiente.

Ortega, P., Galíndez, G., Sühring, S., Rojas, M., Daws, M. \& Pritchard, H. (2011). Seed Germination of Echinopsis schickendantzii (Cactaceae): The Effects of Constant and Alternating Temperatures. Seed Sci Technol, 39, 219-224. Doi: https://doi.org/10.15258/sst.2011.39.1.21

Osorio, C., Alarcón, M., Moreno, C., Bonilla, A., Barrios, J., Garzón, C. \& Duque, C. (2006). Characterization of Odor-active Volatiles in Champa (Campomanesia lineatifolia R. \& P.). J. Agric. Food Chem., 54(2), 509-516. Doi:10.1021 / jf052098c

Parra, A. (2014). Maduración y comportamiento poscosecha de la guayaba (Psidium guajava L.). Una revisión. Revista Colombiana de Ciencias Hortícolas, 8(2), 314-327. Doi: https://doi.org/10.17584/rcch.2014v8i2.3223

Parra, A. \& Fischer, G. (2013). Maduración y comportamiento poscosecha de la feijoa (Acca sellowiana (0. Berg) Burret). Una revisión. Revista Colombiana de Ciencias Hortícolas, 7(1), 98-110. Doi: https://doi.org/10.17584/rcch.2013v7i1.2039

Parra-0., C., Bayly, M., Drinnan, A., Udovicic, F. \& Ladiges, P. (2014). Phylogeny, Major Clades and Infrageneric Classification of Corymbia (Myrtaceae), based on Nuclear Ribosomal DNA and Morphology. Australian Systematic Botany, 22(5), 384-399. Doi: 10.1071 / SB09028

Ranal, M. \& Santana, D. G. (2006). How and why to Measure the Germination Process? Rev. Bras. Bot., 29(1), 1-11. Doi: 10.1590/S0100-84042006000100002

Rego, S., Cosmo, N., Gogosz, A., Kuniyoshi, Y. \& Nogueira, A. (2011). Caracterização morfológica e germinação de sementes de Curitiba prismatica (D. Legrand) Salywon \& Landrum. 
Revista Brasileira de Sementes, Londrina, 33(4), 616-625. Doi:

https://doi.org/10.1590/S0101- 31222011000400003

Salazar, S. A. \& Cancino, G. 0. (2012). Evaluación del efecto de dos suplementos orgánicos en la germinación in vitro de orquídeas nativas de la provincia de Pamplona, Colombia. Revista Colombiana de Biotecnología, 14(1), 53-59. Doi: 10.15446/rev.colomb.biote

Salinas, L. (2016). Efecto alelopático del extracto de semillas de Campomanesia lineatifolia (Myrtaceae) sobre la germinación y crecimiento de maíz y rábano. (Tesis de grado). Programa de Biología, Facultad de Ciencias, Universidad El Bosque, Bogotá.

Savaedi, Z., Parmoon, G., Moosavi, S. A. \& Bakhshande, A. (2019). The Role of Light and Gibberellic Acid on Cardinal Temperatures and Thermal Time Required for Germination of Charnushka (Nigella sativa) Seed. Industrial Crops \& Products, 132, 140-149. Doi: https://doi.org/10.1016/j.indcrop.2019.02.025

Taiz, L., Zeiger, E., Moller I. A. \& Murphy, A. (2017). Fisiologia e desenvolvimento vegetal. (6th ed.). Porto Alegre, Brazil: Artmed.

Takaki, M. (2015). 0 fitocromo e a germinação de sementes de espécies florestais. En F. C. M. Piña, M. B. Figureliolia \& A. Silva (eds.), Ecofisiologia da germinação de sementes. Sementes Florestais: da ecologia à produção. Londrina: Abrates.

Wang, Y. \& Folta, K. M. (2013). Contributions of Green Light to Plant Growth and Development. American Journal of Botany, 100(1), 70-78. Doi: 10.3732/ ajb.1200354

Como Citar: Porras. Y., Pedreros. M., Reyes. W., Balaguera. H. (2020). Efecto de la luz sobre la germinación de semillas champa (Campomanesia lineatifolia R. \& P.). Revista Ciencia y Agricultura. 17(2):23-31 DOl: https//doi.org/10.19053/01228420.v17.n2.2020.10979 